\title{
On mechanisms that enforce complementarity
}

\author{
Berthold-Georg Englert, ${ }^{* \dagger}$ Marlan O. Scully, ${ }^{*}$ and Herbert Walther*§ \\ * Max-Planck-Institut für Quantenoptik, Hans-Kopfermann-Strasse 1, 85748 Garching, Germany \\ ${ }^{\dagger}$ Abteilung für Quantenphysik, Universität Ulm, Albert-Einstein-Allee 1, 89081 Ulm, Germany \\ ${ }^{\ddagger}$ Department of Physics, Texas A $6 M$ University, College Station, TX 77843-4242, USA \\ $\S$ Sektion Physik, Universität München, Am Coulombwall 1, 85748 Garching, Germany
}

(8 October 1999)

\begin{abstract}
In a recent publication Luis and Sánchez-Soto arrive at the conclusion that complementarity is universally enforced by random classical phase kicks. We disagree. One could just as well argue that quantum entanglement is the universal mechanism. Both claims of universality are unjustified, however.
\end{abstract}

PACS numbers: 03.65.Bz, 07.60.Ly, 42.50.Vk

\section{INTRODUCTION AND SUMMARY}

In an interesting recent paper [1], Luis and SánchezSoto reconsidered the loss of fringe visibility in an interferometer with which-way detection devices, and they conclude that this loss occurs because

random classical phase kicks enforce

complementarity always.

We disagree. In particular, the classical-random-phase picture of Ref. [1] does not apply to the thought experiment of a double-slit interferometer for atoms with quantum-optical which-way detection that we introduced in Ref. [2] and treated anew in Ref. [3]. Under the idealized circumstances of a thought experiment, the dynamics is absolutely free of noise and therefore perfectly unitary, and there is no room for any element of classical randomness.

As we shall show here, one could just as well claim that an opposite of (1a) is true, namely that

quantum entanglement enforces complementarity invariably.

In this generality, statements (1a) and (1b) are both excessive, although one could argue, as we show below, that they are mathematically correct in the sense that there are formal procedures that make matters appear either way. There are situations in which (1a) or (1b), or a combination of both, gives an accurate account of the actual physical mechanism that reduces the fringe visibility, but there is no justification for the universality associated with "always" or "invariably."

Rather than "enforce(s) complementarity" one should say more precisely "lead(s) to a loss of fringe visibility" in (1a) and (1 $1 \mathrm{~b})$. Complementarity becomes an issue only when which-way information is made available and wave-particle duality requires a corresponding reduction of the fringe visibility. If truly classical random phase kicks are at work, fringes get lost but nothing can be learned in return about the way through the interferometer, and so the question of how complementarity is enforced doesn't come up. Establishing quantum entanglement is the first step in acquiring which-way knowledge, and then the fringe visibility is accordingly reduced because the "quantum aspects of the which-way detection enforce duality and thus make sure that the principle of complementarity is not circumvented" [4].

\section{CONCERNING THE THOUGHT EXPERIMENT OF REF. [2]}

Let us say a few words about the thought experiment of Ref. [2] before turning to the general, and more abstract, discussion. The crucial quantity is the inner product of $\left|A_{1}\right\rangle$ and $\left|A_{2}\right\rangle$, specified in Eq. (17) of Ref. [1], viz.

$$
\left\langle A_{1} \mid A_{2}\right\rangle=\cos ^{2}(\lambda t)\left\langle 0_{1} 0_{2} \mid 0_{1} 0_{2}\right\rangle+\sin ^{2}(\lambda t)\left\langle 1_{1} 0_{2} \mid 0_{1} 1_{2}\right\rangle,
$$

where $\lambda t$ characterizes the effectiveness of the atomphoton interaction and $\left|0_{1} 1_{2}\right\rangle$, for example, is the state with no photons in cavity $C_{1}$ of Fig. 2 in Ref. [1] and one photon in cavity $C_{2}$. Since $\left|1_{1} 0_{2}\right\rangle$ and $\left|0_{1} 1_{2}\right\rangle$ are orthogonal photon states, we have $\left\langle A_{1} \mid A_{2}\right\rangle=\cos ^{2}(\lambda t)$, of course, and this can be written in the form

$$
\left\langle A_{1} \mid A_{2}\right\rangle=\int d \phi \mathrm{e}^{i \phi} \Omega(\phi),
$$

which seems to suggest an interpretation as a "phase randomization" in the spirit of Ref. [1].

It is true that one can always find a positive weight $\Omega(\phi)$ with all the right properties - the simple choice

$$
\Omega(\phi)=\frac{1}{2 \pi}+\frac{1}{\pi} \cos \phi \cos ^{2}(\lambda t)
$$

serves the purpose if $\cos ^{2}(\lambda t) \leq \frac{1}{2}-$ but the arbitrarily introduced phase variable $\phi$ cannot have any physical significance because, as we said earlier, the idealized thought experiment is free of noise. In fact, as we'll recall in Sec. IIIE below, one can "undo" or reverse the effects of the which-way measurement via quantum erasure, and this is an important difference between the "classical random phase kicks" and the "quantum entanglement" points of 
view. If classical phase noise were at work, quantum erasure couldn't be done.

To emphasize the arbitrariness of the weight $\Omega(\phi)$ in (3) let us show how the overcompleteness of the coherent states $|\alpha\rangle$ [5] (and of states derived from them) can be used to write the key inner-product $\left\langle 1_{1} 0_{2} \mid 0_{1} 1_{2}\right\rangle=0$ of the thought experiment of [2] very easily in the phase-integral form of Ref. [1] . We note the completeness relation (one of many) 6

$$
\mathbb{1}=\int \frac{d \varphi}{2 \pi} \int d I\left[\left(a^{\dagger} a\right) ! I^{-a^{\dagger} a}\right]^{1 / 2}|\alpha\rangle\langle\alpha|\left[\left(a^{\dagger} a\right) ! I^{-a^{\dagger} a}\right]^{1 / 2}
$$

where $\alpha=\sqrt{I} \exp (i \varphi)$ relates the amplitude $\alpha$ of the coherent state to the integration variables $I$ and $\varphi$, and $a$, $a^{\dagger}$ are the ladder operators of the photon mode in question $(a|\alpha\rangle=|\alpha\rangle \alpha)$. Upon using (5) twice and integrating over the intensities $I_{1}$ and $I_{2}$ we get

$$
\begin{aligned}
\left\langle 1_{1} 0_{2}\left|\mathbb{1}_{1} \mathbb{1}_{2}\right| 0_{1} 1_{2}\right\rangle & =\int \frac{d \varphi_{1}}{2 \pi} \int \frac{d \varphi_{2}}{2 \pi} \mathrm{e}^{i\left(\varphi_{1}-\varphi_{2}\right)} \\
& =\int d \phi \mathrm{e}^{i \phi} \frac{1}{2 \pi},
\end{aligned}
$$

where $\phi=\varphi_{1}-\varphi_{2}$ in the last step. Without much effort we have thus arrived at one of the many ways of writing 0 as a phase integral. In view of the obvious arbitrariness of the whole procedure, any physical interpretation of the phase variable $\phi$ is equally arbitrary. Therefore, one should not associate "random classical phase kicks" with the $\phi$ integral.

\section{GENERAL DISCUSSION}

\section{A. Setting the stage}

We turn to the general discussion now. As in [1], we proceed from assuming that, when no which-way detection is attempted, the interferometer prepares the interfering quantum system (photon, electron, atom, ...) in the superposition state $(0<\theta<\pi)$

$$
\left|\psi_{0}\right\rangle=\cos (\theta / 2)\left|\psi_{1}\right\rangle+\mathrm{e}^{i \phi} \sin (\theta / 2)\left|\psi_{2}\right\rangle,
$$

where $\left|\psi_{1}\right\rangle$ and $\left|\psi_{2}\right\rangle$ symbolize the basic alternatives, i.e. the ways, of the interferometer — such as "through this slit" and "through that slit" in a Young's double-slit experiment. A measurement of the probability for finding the system in the $\varphi$ dependent state

$$
|\psi(\varphi)\rangle=\frac{1}{\sqrt{2}}\left(\left|\psi_{1}\right\rangle+\mathrm{e}^{i \varphi}\left|\psi_{2}\right\rangle\right)
$$

produces the interference pattern

$$
p_{0}(\varphi)=\left|\left\langle\psi(\varphi) \mid \psi_{0}\right\rangle\right|^{2}=\frac{1}{2}[1+\sin \theta \cos (\varphi-\phi)],
$$

so that $\mathcal{V}_{0}=\sin \theta$ is the fringe visibility.

Now, an attempt to gain which-way knowledge is made (or any other manipulation of the interferometric setup). In concordance with Ref. [1], we shall take for granted that the probabilities for finding the basic states $\left|\psi_{1}\right\rangle$ and $\left|\psi_{2}\right\rangle$ are not affected. Therefore, the net effect can only be a change of the interference pattern from $p_{0}(\varphi)$ of (9) to

$$
p_{\epsilon}(\varphi)=\frac{1}{2}\left[1+|\epsilon| \sin \theta \cos \left(\varphi-\phi-\delta_{\epsilon}\right)\right],
$$

where the complex parameter $\epsilon=|\epsilon| \exp \left(i \delta_{\epsilon}\right)$ is subject to the restriction $|\epsilon| \leq 1$. As a consequence, the fringe pattern is shifted by $\delta_{\epsilon}$ and its visibility is reduced to $\mathcal{V}=|\epsilon| \mathcal{V}_{0}$.

The transition from (9) to (10) means that the statistical operator of the quantum system has undergone the transformation

$$
\begin{aligned}
\rho_{0}= & \left|\psi_{0}\right\rangle\left\langle\psi_{0}\right| \\
\rightarrow \rho_{\epsilon}= & \left|\psi_{1}\right\rangle \cos ^{2}(\theta / 2)\left\langle\psi_{1}|+| \psi_{2}\right\rangle \sin ^{2}(\theta / 2)\left\langle\psi_{2}\right| \\
& +\left|\psi_{1}\right\rangle \cos (\theta / 2) \mathrm{e}^{-i \phi} \epsilon^{*} \sin (\theta / 2)\left\langle\psi_{2}\right| \\
& +\left|\psi_{2}\right\rangle \sin (\theta / 2) \mathrm{e}^{i \phi} \epsilon \cos (\theta / 2)\left\langle\psi_{1}\right|
\end{aligned}
$$

which is nonunitary if $|\epsilon|<1$.

\section{B. The "classical random phase kicks" argument}

The stage is now set for a presentation of the "classical random phase kicks" argument of Ref. [1]. It has essentially two ingredients. (i) First, an actual classical randomization of the phase $\phi$ in (7), such that additional phase factors $\exp \left(i \phi^{\prime}\right)$ with $\phi^{\prime}$ in the range $\phi^{\prime} \cdots \phi^{\prime}+d \phi^{\prime}$ occur with probability $d \phi^{\prime} \Omega\left(\phi^{\prime}\right)$, would cause the transition (11) with $\epsilon$ given by $[7$

$$
\epsilon=\int d \phi^{\prime} \Omega\left(\phi^{\prime}\right) \mathrm{e}^{i \phi^{\prime}}
$$

provided that the positive weight $\Omega\left(\phi^{\prime}\right)$ is normalized in accordance with

$$
\int d \phi^{\prime} \Omega\left(\phi^{\prime}\right)=1
$$

(ii) Second, any given value of $\epsilon$, in particular the one needed in (10), can be written in the form (12) with a suitably chosen $\Omega\left(\phi^{\prime}\right)$. There is, of course, an abundance of permissible $\Omega$-weights, of which

$$
\Omega\left(\phi^{\prime}\right)=\frac{1+|\epsilon|}{2} \delta\left(\phi^{\prime}-\delta_{\epsilon}\right)+\frac{1-|\epsilon|}{2} \delta\left(\phi^{\prime}-\delta_{\epsilon}-\pi\right)
$$

and

$$
\Omega\left(\phi^{\prime}\right)=\left\{\begin{array}{cl}
(2 \chi)^{-1} & \text { for }\left|\phi^{\prime}-\delta_{\epsilon}\right| \leq \chi \leq \pi \\
& \text { with }|\epsilon|=\chi^{-1} \sin \chi \\
0 & \text { elsewhere }
\end{array}\right.
$$


are perhaps the simplest ones; another explicit example is given in Eq. (12) of [1].

Since there is always a $\Omega$-weight with the desired properties, one can justifiably state that the net effect is always as if a classical randomization of the phase $\phi$ of (7) had occurred. According to Ref. [1], however, this is the actual mechanism, notwithstanding the said as-if character and irrespective of what is really going on.

We disagree with this physical interpretation of the mathematical fact (ii) — and thus with the central message of Ref. [1]. The as-if aspect is relevant and deserves emphasis.

\section{The "quantum entanglement" argument}

Before offering further remarks, it is expedient to put the "quantum entanglement" argument on record, which has also two logical ingredients. (i') First, an interaction of the system with its environment (part of which might be a which-way detector controlled by the experimenter) establishes an entangled state [8]

$$
\begin{aligned}
& \left(\left|\psi_{1}\right\rangle \cos ^{2}(\theta / 2)\left\langle\psi_{1}\right|\right) \otimes U_{\mathrm{env}} \rho_{\mathrm{env}} U_{\mathrm{env}}^{\dagger} \\
+ & \left(\left|\psi_{2}\right\rangle \sin ^{2}(\theta / 2)\left\langle\psi_{2}\right|\right) \otimes \rho_{\mathrm{env}} \\
+ & \left(\left|\psi_{1}\right\rangle \cos (\theta / 2) \mathrm{e}^{-i \phi} \sin (\theta / 2)\left\langle\psi_{2}\right|\right) \otimes \rho_{\mathrm{env}} \mathrm{U}_{\mathrm{env}}^{\dagger} \\
+ & \left(\left|\psi_{2}\right\rangle \sin (\theta / 2) \mathrm{e}^{i \phi} \cos (\theta / 2)\left\langle\psi_{1}\right|\right) \otimes \mathrm{U}_{\mathrm{env}} \rho_{\mathrm{env}}
\end{aligned}
$$

and consequently effects a transition of the form (11) with

$$
\epsilon=\operatorname{tr}_{\mathrm{env}}\left\{U_{\mathrm{env}} \rho_{\mathrm{env}}\right\}
$$

where $\rho_{\text {env }}$ is a statistical operator of the environment prior to the interaction, $U_{\mathrm{env}}$ is a unitary operator acting solely on the environment variables, and $\operatorname{tr}_{\text {env }}\{\cdots\}$ is the injunction to trace over these variables. (ii') Second, any given value of $\epsilon$, in particular the one needed in (10), can be written in the form (17) with suitably chosen $\rho_{\text {env }}$ and $U_{\text {env }}$. Here, too, we have an abundance of possible choices. A specific example is a harmonic oscillator with

$$
\rho_{\mathrm{env}}=|0\rangle\langle 0|
$$

and $U_{\text {env }}$ such that

$$
\begin{aligned}
& U_{\text {env }}|0\rangle=\epsilon|0\rangle+\mathrm{e}^{i \delta_{\epsilon}} \sqrt{1-|\epsilon|^{2}}|1\rangle, \\
& U_{\text {env }}|1\rangle=\mathrm{e}^{i \delta_{\epsilon}} \sqrt{1-|\epsilon|^{2}}|0\rangle-\epsilon|1\rangle,
\end{aligned}
$$

where $|0\rangle$ and $|1\rangle$ are the ground and first excited oscillator states, respectively.

Since there is always a pair $\rho_{\text {env }}, U_{\text {env }}$ with the desired properties, one can justifiably state that it is always as if a quantum entanglement had caused the transition (11). But, of course, one cannot disregard its as-if character and conclude that this mechanism is invariably the actual one, irrespective of what is really going on.

\section{SAI equivalence}

In Ref. [9], Stern, Aharonov, and Imry (SAI) largely anticipated the formal aspects of Ref. [1] without, however, arriving at the questionable statement (1a). Moreover, SAI have noted that one can turn the "quantum entanglement" picture into the "classical random phase kicks" one by summing over the eigenstates of $U_{\text {env }}$ when evaluating the trace in (17). Likewise, for a given $\Omega\left(\phi^{\prime}\right)$ one can always construct a corresponding $\rho_{\mathrm{env}}, U_{\text {env }}$ pair. The two as-if descriptions are, so to say, "SAI equivalent." In the example of Eqs. (18) and (19) we have

$$
U_{\mathrm{env}}| \pm\rangle= \pm \mathrm{e}^{i \delta_{\epsilon}}| \pm\rangle
$$

with

$$
| \pm\rangle=\sqrt{(1 \pm|\epsilon|) / 2}|0\rangle \pm \sqrt{(1 \mp|\epsilon|) / 2}|1\rangle
$$

so that the phases $\delta_{\epsilon}$ and $\delta_{\epsilon}+\pi$ have the respective weights $(1+|\epsilon|) / 2$ and $(1-|\epsilon|) / 2$, and the $\Omega\left(\phi^{\prime}\right)$ example of (14) obtains. Put frivolously, their SAI equivalence shows that the two seemingly far-reaching conclusions (1a) and (1b) are both in fact just far-fetched.

It seems that the authors of Ref. [1] regard the SAI equivalence of the two as-if accounts as evidence in favor of the universality they attribute to their "classical random phase kicks" picture. In fact, this wouldn't do justice to the enormous differences in the actual physical situations. Despite the formal SAI equivalence of (12) $-(14)$ and $(17)-(19)$, the physical significance of the phases $\delta_{\epsilon}$ and $\delta_{\epsilon}+\pi$ in (14) is utterly different from the one in $(20)$.

\section{E. Quantum erasure}

If the transition (11) is actually the result of classical phase kicks that are truly random, then the value of $\epsilon$ is all the experimenter can ever know. In particular, he cannot determine the $\Omega$-weight of (12) and (13) because the random nature of the phase kicks precludes any further knowledge 10].

In marked contrast, much information is potentially available to the experimenter if quantum entanglement causes the transition (11), in particular the entanglement with a well controlled which-way detection device. For instance, the experimenter could perform a judiciously chosen measurement on the final state of the device to learn about the way taken. More relevant for the present discussion is the possibility of quantum erasure [11, 3]: The experimenter sorts the interfering quantum systems into subensembles in accordance with the outcome of a measurement that determines in which one of the $U_{\text {env }}$ eigenstates the which-way detector is found. In the example of (18) and (19), the observable $|+\rangle\langle+|-|-\rangle\langle-|$ would be measured, for instance, and the outcomes \pm 1 found with relative frequencies $(1 \pm|\epsilon|) / 2$. 
The corresponding subensembles have statistical operators that project to

$$
\left|\psi^{( \pm)}\right\rangle=\cos (\theta / 2)\left|\psi_{1}\right\rangle \pm \mathrm{e}^{i\left(\phi+\delta_{\epsilon}\right)} \sin (\theta / 2)\left|\psi_{2}\right\rangle
$$

and each subensemble exhibits fringes with the original visibility $\mathcal{V}_{0}=\sin \theta$

If he'd wish to do so, the experimenter could then say that it is as if the interaction had created two subensembles by the transitions $\phi \rightarrow \phi+\delta_{\epsilon}$ and $\phi \rightarrow \phi+\delta_{\epsilon}+\pi$ and allocated the fractions $(1 \pm|\epsilon|) / 2$ to them. In this manner, the experimenter would actually determine $\Omega\left(\phi^{\prime}\right)$ of (14), and we emphasize that he has this possibility only if the quantum-entanglement mechanism is actually at work. Quantum erasure is impossible if the interfering system has suffered from truly random phase kicks. Hence, the $\Omega$-weight has a phenomenological significance only if the "quantum entanglement" picture is actual, but not when the "classical random phase kicks" are in action.

But, again there is an essential as-if character to this "interaction creates subensembles" notion. A measurement of another observable, such as $|0\rangle\langle 0|-| 1\rangle\langle 1|$, for instance, would result in a different pair of subensembles with an equally justified as-if interpretation. One of the subensembles may yield fringes with a visibility $\mathcal{V}_{\text {sub }}$ that exceeds $\mathcal{V}_{0}=\sin \theta$, even $\mathcal{V}_{\text {sub }}=1$ is achievable - an impossible feat if (11) resulted from "classical random phase kicks."

The quantum-erasure sorting into the subensembles of (22) labels each of the interfering quantum systems by the phase $+\left(\phi+\delta_{\epsilon}\right)$ or by the phase $-\left(\phi+\delta_{\epsilon}\right)$, and as a consequence of the quantum-mechanical indeterminacy, these labels are attached randomly. This invites the question: 'Aren't these labels just the classical random phases of Ref. [1]?' Answer: No, they aren't, because the randomness of the labeling process is of a purely quantum nature and has absolutely nothing to do with classical phase noise.

\section{RELEVANCE FOR REAL-LIFE EXPERIMENTS}

In closing, we'd like to remark on the relevance of these somewhat abstract considerations for real-life laboratory experiments. In any interferometer experiment, there are unavoidably parameters that cannot be fully controlled, or maybe not at all. For example, the beam splitters and mirrors of a Mach-Zehnder interferometer could be subject to erratic mechanical vibrations, and the resulting loss of fringe visibility would be correctly blamed on "classical random phase kicks." Similarly, optical elements of modern atom interferometers 12 employ laser beams, and their jitters give rise to random phases. Deliberately introduced quantum entanglement comes in addition, so that a satisfactory description of an experiment must take both mechanisms for visibility loss into account.
The recent atom interferometer experiments by Dürr, Nonn, and Rempe (DNR) 13 illustrate these matters quite well. DNR had to be content with a maximal fringe visibility of $\sim 70 \%$ although their interferometer is symmetric $\left[\cos ^{2}(\theta / 2) \simeq \sin ^{2}(\theta / 2) \simeq \frac{1}{2}, \sin \theta \lesssim 1\right]$. Clearly, lack of control over some parameters - the hallmark of random noise - is responsible for the $\sim 30 \%$ reduction. The which-way detection performed by DNR led to a complete loss of fringe visibility - undoubtedly the result of quantum entanglement, as demonstrated by the resurrection of the fringes with the aid of quantum erasure.

[1] A. Luis and L. L. Sánchez-Soto, Phys. Rev. Lett. 81, 4031 (1998).

[2] M. O. Scully, B.-G. Englert, and H. Walther, Nature (London) 351, 111 (1991).

[3] B.-G. Englert, M. O. Scully, and H. Walther, Am. J. Phys. 67, 325 (1999).

[4] B.-G. Englert, Phys. Rev. Lett. 77, 2154 (1996).

[5] See any textbook on quantum optics, such as M. O. Scully and M. S. Zubairy, Quantum optics (Cambridge University Press, Cambridge, 1997).

[6] Incidentally we note that, for all values of the phase parameter $\varphi$, the $I$ integration yields the projector to the corresponding London's phase states [F. London, Z. Phys. 37, 915 (1926); 40, 193 (1927)].

[7] For simplicity we don't consider the additional possibility of randomizing the angle parameter $\theta$ in (ब).

[8] More general possibilities can be considered; for the sake of simplicity, we are content with (16).

[9] A. Stern, Y. Aharonov, and Y. Imry, Phys. Rev. A 41, 3436 (1990).

[10] In Ref. 11 the interference pattern (10) is regarded as $(\pi$ times) a probability distribution for the phase variable $\varphi$, and then associated with a phase dispersion. This, however, has no bearing on the issue at hand, since $p_{\epsilon}(\varphi)$ contains no information about $\Omega\left(\phi^{\prime}\right)$ beyond what is stated in Eqs. (12) and 13.

[11] M. O. Scully and K. Drühl, Phys. Rev. A 25, 2208 (1982).

[12] Atom Interferometry, edited by P. Berman (Academic, New York, 1997).

[13] S. Dürr, T. Nonn, and G. Rempe, Nature (London) 395, 33 (1998); Phys. Rev. Lett. 81, 5705 (1998). 